\title{
Alcohol consumption in domestic and wild rats'
}

ERHARD O. EIMER AND R. J. SENTER UNIVERSITY OF CINCINNATI
A group of domestic rats and a group of wild pack rats were exposed to food and a $20 \%$ alcohol solution continuously for 20 weeks. Subsequently, both groups were exposed to food, alcohol solution, and water for nine weeks. No major changes in alcohol consumption rates occurred in either group within either of the two periods. Alcohol consumption decreased for both groups when the choice situation (alcohol vi water) was instituted. This decrease was more pronounced for domestic rats than for wild rats. The results are discussed in terms of potential physiological or other pre-experimental factors affecting alcohol consumption rate.

Experimental attempts to establish in laboratory rats a behavior pattern analogous to human alcoholism have generally reaped negative results. Temporary increases in alcohol consumption have been achieved repeatedly (Senter \& Sinclair, 1967; Senter, Smith, \& Lewin, 1967; Myers, 1966), but the establishment of chronic, autonomous, maladaptive alcohol consumption is so rare that McConnell \& Clay (1963) have questioned whether mammals lower than man are susceptible to the development of such behavior patterns. An exception to this trend has been reported by Richter (1957). While this researcher reported repeated failure to addict domestic laboratory rats, he succeeded in "addicting two fierce, aggressive wild rats trapped from the streets. This was done by restricting fluid intakes to a $20 \%$ solution of alcohol over a period of six months. When given a choice of food, water and a $20 \%$ solution of alcohol, these rats gradually ate less food, drank less plain water, and more alcohol. As a result they progressively lost weight and finally died" (p. 218). The present research was undertaken to investigate more systematically the observation made by Richter.

Method

Twenty wild pack rats (Genus Neotoma), captured in Arizona, and 16 domestic hooded rats were continuously exposed to standard laboratory rat chow and a $20 \%(\mathrm{~V} / \mathrm{V})$ alcohol solution for 20 weeks. Subsequent to this exposure period, both groups were given rat chow, a $20 \%$ alcohol solution, and water for nine weeks. Consumption of alcohol solution was recorded daily throughout the 20 week exposure period and the nine week choice period. Weekly mean consumption of alcohol solution in $\mathrm{ml}$ for each $\mathrm{S}$ was established for data analysis.

\section{Resulits}

Richter's (1957) finding was not replicated. An analysis of variance on exposure-period data indicates that throughout the exposure period domestic rats consumed more alcohol than did wild rats $(F=367.86$, $\mathrm{df}=1 / 34, \quad \mathrm{p}<.01$ ), a finding that is not surprising since the domestic rats were heavier than were the wild rats $(257 \mathrm{~g}$ vs $199 \mathrm{~g})$. A significant overall increase in alcohol consumption over this period was observed ( $F$ for linear trend of time main effect $=17.41, \mathrm{df}=1 / 646, \mathrm{p}<.01)$. A trend analysis on the groups by time interaction, however, indicates that this linear increase is entirely accounted for by the domestic rats $(F=34.98, d f=1 / 646, p<.01)$. If any linear change occurred in the group of wild rats at all, it was a decrease in alcohol consumption. (See Fig. 1)

A second analysis was performed in which, for each rat, mean overall alcohol consumption for the entire exposure period and mean overall alcohol consumption for the entire choice period were considered the dependent variables. The between-groups effect was nonsignificant $(F=1.253, d f=1 / 34)$, although even a significant variance ratio would have been inconsequential in this case. As expected, both groups consumed less alcohol during the choice period, when they had water available, than during the exposure period ( $F$ $=237.00, \mathrm{df}=1 / 34, \mathrm{p}<.01$ ). A surprising finding was made in the observation that alcohol consumption

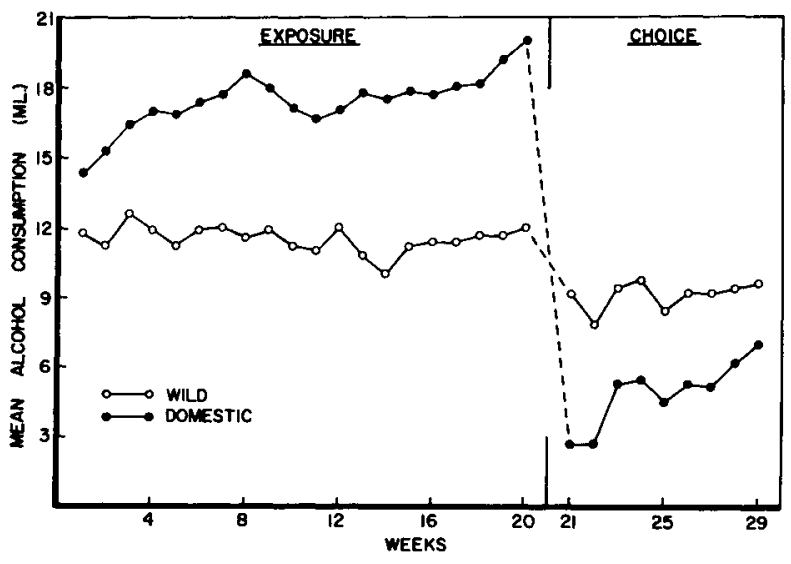

Fig. 1. Daily mean consumption of $20 \%$ alcohol solution, averaged over weeks, by domestic and wild rats over a 20 week exposure period and a nine week choice period. 
decreased considerably less for wild rats than for domestic rats (Interaction $\mathrm{F}=99.87, \mathrm{df}=1 / 34, \mathrm{p}<.01$; see also Fig. 1).

\section{Discussion}

In view of the major experimental hypothesis, the present experiment adds to the already long list of studies in which an alcoholic syndrome has not been established in rats. In the course of the exposure period, several animals (not included in the analysis), both domestic and wild rats, died of middle ear infection and similar diseases. The deaths were not preceded by less water consumption (since there was no water available) or any maladaptive, excessive drinking behavior. In fact, deaths occurred largely in animals consuming relatively little alcohol. The only possibility of linking death to alcohol consumption in the present study seems to be that some animals may have become dehydrated due to minimal liquid ingestion, possibly because the $20 \%$ solution may have been too aversive (Myers, 1966).

At no time during the experiment was a systematic and pronounced increase in alcohol consumption observed in any Ss, a finding contrary to that of Richter (1957). The gradual overall increase in alcohol ingestion among domestic rats during the exposure period, although statistically significant, may be considered as quantitatively meaningless since it is small enough to be accounted for in terms of changes in body weight. At the termination of the experiment, all animals appeared healthy. As has been observed and reported previously (Senter, Eimer, \& Richman, 1968), large individual differences in alcohol consumption between animals, and a high stability of drinking rate within animals over time, were observed.

The finding of major interest is the observation that alcohol consumption rate decreased less for wild rats than for domestic rats when both groups were introduced to the choice situation in which both alcohol and water were available, and that, in fact, the wild rats (still having the lower body weight), then consumed more alcohol than did domestic rats. This finding is not in complete contradiction to Richter's (1957) finding. An interpretation of this result has to be tentative, but it may be sought in the fact that wild rats, in contrast to domestic rats, have large adrenal glands. The adrenal glands generate adrenal-cortical steroids, one of which is alcohol dehydrogenase (ADH), which oxidizes alcohol in the liver (von Wartburg \& Papenberg, 1966). It is conceivable that during the exposure phase of the experiment, alcohol ingestion in the wild rats was limited only by their liquid requirement, and that these animals, due to the size of their adrenal glands, are capable of metabolizing ethanol at a higher rate than domestic rats could. This interpretation would lead to the notion that rate of alcohol ingestion is related to, and possibly controlled by, the physiological capacity of the organism for disposing of ethanol. This tentative interpretation corresponds well with the findings (Senter, Eimer, \& Richman, 1968) of large individual differences in consumption rate, which, since they are of preexperimental origin, may well be physiological in nature. This conclusion may be somewhat disturbing since it is quite inconsistent with many "learning" hypotheses concerning human alcoholism. It may be said in defense of this hypothesis, however, that while it may account for differences in alcohol consumption rate, there is at present no need for it to account for alcoholism, since this syndrome has not been produced in the present study.

\section{References}

McCONNEL, J. V., \& CLAY, MARGARET. Conditions affecting voluntary alcohol consumption in laboratory rats. Abstract. Report by the Scientific Advisory Council on the Alcoholism Research Grant Program, Licensed Beverage Industries, New York, 1963.

MYERS, R. D. Voluntary alcohol consumption in animals: Peripheral and intracerebral factors. Psychosom. Med., 1966, 28, 484-497.

RICHTER, C. P. Decreased appetite for alcohol and alcoholic beverages produced in rats by thyroid treatment. In H. Hoagland (Ed.), Hormones, brain function, and behavior. Academic Press: New York, 1957.

SENTER, R. J., EIMER, E. O., \& RICHMAN, C. L. Intersubject and intrasubject variability in the consumption of alcohol. Psychon. Sci. in press.

SENTER, R. J., \& SINCLAIR, J. D. Self-maintenance of intoxication in the rat: A modified replication. Psychon. Sci., 1967, 9, 291-292.

SENTER, R. J., SMITH, F. W., \& LEWIN, S. Ethanol ingestion as an operant response. Psychon. Sci., 1967, 8, 291-292.

VON WARTBURG, J. P., \& PAPENBERG, J. Alcohol dehydrogenase in ethanol metabolism. Psychosom. Med., 1966, 28, 405-413.

Note

1. This research was supported by USPHS Grant No. MH 11895-01. 